\title{
Glottopol
}

Revue de sociolinguistique en ligne

$34 \mid 2020$

Les « langues de France ", 20 ans après

\section{Hommages à Jean Le Dû}

Philippe Blanchet, Guylaine Brun-Trigaud, Ronan Calvez, Gudrun Ledegen, Malo Morvan, René Tabouret-Keller, Mannaig Thomas et Nelly Blanchard

\section{(2) OpenEdition}

Journals

Édition électronique

URL : https://journals.openedition.org/glottopol/335

DOI : 10.4000/glottopol.335

ISSN : 1769-7425

Éditeur

Presses universitaires de Rouen et du Havre

\section{Référence électronique}

Philippe Blanchet, Guylaine Brun-Trigaud, Ronan Calvez, Gudrun Ledegen, Malo Morvan, René

Tabouret-Keller, Mannaig Thomas et Nelly Blanchard, « Hommages à Jean Le Dû », Glottopol [En ligne],

34 | 2020, mis en ligne le 01 juillet 2020, consulté le 02 octobre 2021. URL : http://

journals.openedition.org/glottopol/335; DOI : https://doi.org/10.4000/glottopol.335 


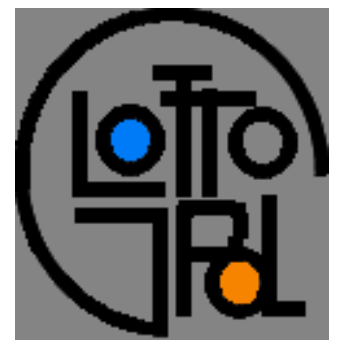

\section{GLOTTOPOL}

Revue de sociolinguistique en ligne $\mathrm{n}^{\circ} 34$ - juillet 2020

Les "langues de France " : 20 ans après

\section{SOMMAIRE}

Hommages à Jean Le Dî

Christian Lagarde : "Langues de France ». Au-delà du symbolique?

Entretien avec Bernard Cerquiglini, par Christian Lagarde.

Entretien avec Paul de Sinety, Délégué général à la langue française et aux langues de France, par Christian Lagarde.

Georg Kremnitz : La problématique initiale de la liste Cerquiglini et ses effets ultérieurs.

Alain Viaut : De "langue régionale » à "langue de France » ou les ombres du territoire.

Wanda Mastor: Le statut constitutionnel des langues régionales en droit comparé. De la reconnaissance à l'indifférence.

Philippe Martel, Marie-Jeanne Verny: Les langues régionales au Parlement, ou l'éternel retour.

Romain Colonna : Les «langues de France »: des langues non-étatiques au pays de l'Étatnation.

Hervé le Bihan : La langue bretonne : une visibilité toute en retenue.

Véronique Bertile : Les langues d'outre-mer : des langues de France? Approche juridique.

Jacques Vernaudon: Les langues polynésiennes et kanak, des "langues de France» en contexte de décolonisation.

Luc Biichlé : Qu'advient-il de l'arabe de France? Mise en perspective sociolinguistique...

Pascal Ottavi : L'épervier, la cage et le passereau.

Marielle Rispail : Le francique lorrain, langue de France? Réflexions et témoignages.

\section{Compte rendu de lecture}

Par Salih Akin : Jean Le Dû \& Yves Le Berre, Métamorphoses. Trente ans de sociolinguistique à Brest (1984-2014), Brest, Centre de Recherche Bretonne, 2019, 302 p.

http://glottopol.univ-rouen.fr 


\section{HOMMAGES À JEAN LE DÛ ${ }^{1}$}

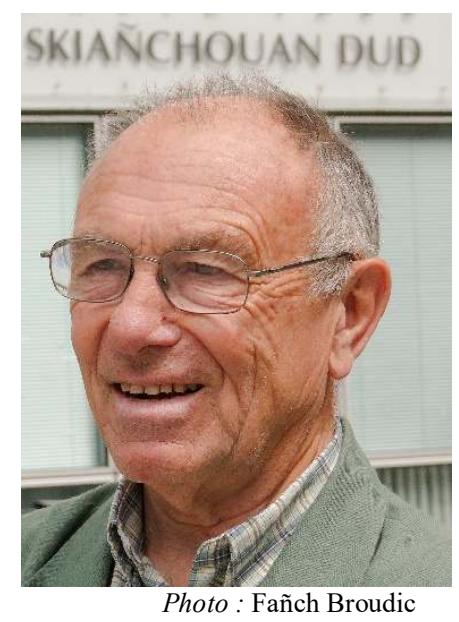

\section{Philippe Blanchet Université de Rennes 2}

Sociolinguiste breton et spécialiste de la langue bretonne bien connu, il était professeur émérite à l'Université de Bretagne Occidentale (Brest) et s'intéressait aussi à de nombreuses situations sociolinguistiques. Il avait soutenu, dès nos premiers travaux, l'émergence d'une équipe de sociolinguistique à Rennes 2, en participant à notre première journée d'étude en 1994. C'est de là qu'est né le premier numéro des Cahiers de Sociolinguistique intitulé « Langues et parlers de l'Ouest », paru en 1996 sous les auspices de Francis Manzano. Il était encore venu il y a 2 ou 3 ans nous présenter son atlas linguistique des Antilles.

Jean était aussi un bon et joyeux camarade avec qui j'ai partagé beaucoup de bons moments, et pas qu'en Bretagne, surtout quand il faisait binôme avec son alter ego, Yves Le Berre, que je salue bien amicalement et solidairement. Jean m'appelait « le moko » sobriquet que les marins bretons donnent aux marins provençaux. En retour je l'appelais «Yann-Fañch » pour le faire un peu bisquer...

C'est pour lui dire merci braz publiquement que nous partageons notre tristesse rennaise avec la communauté des sociolinguistes francophones et tout particulièrement avec nos collègues et ami.e.s de Brest.

\footnotetext{
${ }^{1}$ Certains de ces hommages ont circulé sur la liste du Réseau francophone de sociolinguistique, ainsi que sur le blog www.langue-bretonne.org, tenu par Fañch Broudic. Leurs auteurs ont accepté que leur texte soit repris ici, avec d'autres hommages recueillis suite à la proposition de publication de la rédaction de Glottopol. Leur présence dans ce numéro sur Les langues de France s'imposait ; Jean Le Dû figure d'ailleurs dans son comité de lecture, et Salih Akin y rend compte de son dernier ouvrage avec Y. Le Berre, comme il le lui avait proposé.
} 
Guylaine Brun-Trigaud ${ }^{2}$

\section{Université de Nice-Côte d'Azur, laboratoire CNRS Bases, corpus, langage}

La nouvelle de la disparition de Jean m'a fait l'effet d'une chute dans le vide... Puis une immense tristesse a fait place à l'incrédulité : Jean n'était plus là, il était parti sans dire «Salut! ", comme il le faisait à la fin de chacun de nos fréquents appels téléphoniques. C'était le point final de près de trente ans de travaux en commun et de complicité.

\section{Premières rencontres}

J'ai croisé Jean pour la première fois en mars 1987 à Paris, lors d'une intervention dans le cours de dialectologie des Hautes Études alors que je préparais ma thèse.

En 1989, il devint directeur du GRECO (Groupe de Recherches Coordonnées) des Atlas linguistiques régionaux et il se retrouva à la tête d'une entreprise qui avait reçu l'injonction de prendre le virage de l'informatisation, et, il faut le dire, cela s'est avéré plus difficile que prévu. Deux chantiers étaient alors en cours : la saisie des données pour la poursuite de la publication des atlas et la confection d'un index regroupant la totalité de leurs données lemmatisées.

En 1993, dans un courrier adressé à l'ensemble des participants du GRECO et aux vacataires, dont je faisais partie à l'époque, il faisait le constat du « manque de bras » pour mener à bien ces deux missions : je lui proposais alors les miens. Aussitôt, il me confia la coordination sur les index et cela fut le début d'une collaboration qui s'avéra longue et fructueuse.

\section{Ma découverte des cartes de François Falc'hun}

Ainsi, lors d'un colloque à Brest en 1997, Jean me fit découvrir un trésor conservé précieusement au CRBC : 2000 cartes manuscrites confectionnées par l'abbé Falc'hun à partir de l'Atlas linguistique de la France. C'était le début de l'aventure qui nous mena, avec Yves Le Berre, aux Lectures de l'Atlas linguistique de la France paru au CTHS (Comité des travaux historiques et scientifiques) en 2005, pour lequel j'ai réalisé plus de 800 cartes dans l'esprit initial de celles de Falc'hun.

\section{Sa rencontre avec un certain William Labov}

C'est d'ailleurs cet ouvrage que Jean a glissé à un certain William Labov alors que nous participions à un colloque à Lyon en 2009 (photo ci-dessus), ce qui lui valut d'être invité à Philadelphie au domicile de W. Labov, pendant l'un de ses séjours aux États-Unis.

\section{L'Atlas des Petites Antilles}

À la même époque, Jean m'apprit qu'il avait saisi les données pour un atlas créole dont il avait dirigé les enquêtes et qu'il avait besoin de conseils pour faire les cartes... Évidemment, de fil en aiguille, je me suis retrouvée « embarquée » dans cette nouvelle aventure qui nous conduisit à la publication des deux volumes de l'Atlas linguistique des Petites Antilles (CTHS 2011 et 2013).

\section{Nos derniers travaux en commun}

Nous avons écrit plusieurs contributions ensemble (une bonne quinzaine), et notamment la synthèse romane des dénominations de la pie, dans le cadre de l'Atlas linguistique roman, dont les membres s'associent tous à notre peine aujourd'hui.

\footnotetext{
${ }^{2}$ Ce texte a été écrit pour être publié sur le blog www.langue-bretonne.org, tenu par Fañch Broudic.
} 
Enfin, ces derniers temps, après m'avoir conseillé pour la rédaction de mon dernier livre sur Les parlers de la Creuse, il avait commencé la préparation d'un ouvrage sur le breton, fruit de ses réflexions sur un thème qui lui tenait tant à cœur et qui aurait été illustré de cartes synthétisées à partir de NALBB (Nouvel atlas linguistique de la Basse-Bretagne). Hélas, ce travail ne verra pas le jour.

Plus qu'un collègue, plus qu'un modèle (il n'aurait pas aimé le mot «maître »), je déplore la perte d'un ami très cher, toujours à l'écoute et soucieux des autres, toujours ouvert à une discussion sérieuse ou plus légère, et surtout plein d'une expérience irremplaçable.

«Salut Jean!»

Ronan Calvez

\section{CRBC (EA 4451 / UMS 3554) \& Université de Bretagne occidentale (Brest)}

Durant sa promenade quotidienne, Jean Le Dû est décédé brutalement aux côtés de son épouse Françoise. Je voudrais saluer le professeur et l'homme, fraternellement. Et je me fais ici le porte-parole d'Annie, de Mannaig, de Nelly, d'Yves et de tous les collègues du Centre de Recherche Bretonne et Celtique (le CRBC).

Jean était professeur émérite de breton \& celtique de l'université de Brest, et membre du CRBC depuis les origines du laboratoire. En 1984, en compagnie d'Yves Le Berre, il avait fondé le Groupe de Recherche sur l'Economie Linguistique de la Bretagne. Tous deux ont mis en œuvre et animé un séminaire pluriannuel, carrefour intellectuel incontournable où se sont croisés et où ont débattu dialectologues, linguistes, sociolinguistes et littéraires du monde entier. Les actes de ces séminaires ont été rassemblés au sein de la revue La Bretagne linguistique, qui continue de paraître. En effet, grâce à Mannaig et Nelly, le séminaire continue et continuera de permettre de débattre sur les pratiques linguistiques en Bretagne, au regard d'autres pratiques comparables dans le monde ou de savoirs issus d'autres sciences.

Le passage, au mois de juin, à la publication numérique sur OpenEdition Journals de la revue La Bretagne Linguistique réjouissait Jean, qui y voyait une nouvelle étape dans la vie de ce séminaire et de cette publication. Le prochain numéro - le $n^{\circ} 23$-, auquel il a contribué, tout premier numéro en ligne de la revue, lui sera dédié. Lorsque j'ai annoncé la mort de Jean à Hélène, secrétaire d'édition au CRBC, elle m'a confié qu'elle finissait, le matin même, de mettre en page son article pour ce numéro en ligne de la Bretagne Linguistique. Je trouve que ce clin d'œil du destin est triste, mais je trouve aussi qu'il est joli.

Les recherches de Jean, très fin connaisseur du breton et de ses variétés, portaient sur la géographie linguistique et la sociolinguistique. Son grand œuvre reste et restera le Nouvel Atlas Linguistique de la Basse-Bretagne, publié en 2001, fruit d'un long travail de collecte attentive et respectueuse auprès des bretonnants. De même, son dictionnaire du breton de Plougrescant, publié en 2012, témoigne de son amour pour le breton des siens - de sa mère notamment. Enfin, en 2019, il aura eu le plaisir de voir ses articles de sociolinguistique, coécrits avec Yves, publiés sous le titre Métamorphoses.

Ar re o deuz bet kenteliou gantañ a zalho soñj euz ar helenner lemm e spered, ha tomm e galon ouz brezoneg e dud hag ouz an oll vrezonegou. Gouzoud a oueze ervad petra zinifie ha petra zinifi kaozeal brezoneg. Kar Jean a oueze ervad petra zinifi kaozeal, petra zinifi komz.

Jean n'était pas qu'un excellent bretonnant. Il était polyglotte et pratiquait aussi avec bonheur le gaélique d'Irlande, l'anglais, l'allemand, l'espagnol, le turc, le néerlandais ou encore le créole. Les langues étaient pour lui un moyen de tisser des liens, d'échanger et de faire des rencontres. Tisser des liens, échanger et faire des rencontres : c'est aussi cela que je garderai comme héritage de Jean. La nature n'existe pas et, seules, les cultures existent. 
Gudrun Ledegen

Université de Rennes 2

J'ai eu l'immense plaisir de travailler avec Jean ces dernières années sur un autre aspect de ses connaissances insondables moins connu : il pratiquait très bien le flamand, ayant vécu vers le Westhoek, et avait partagé une longue amitié et un commun intérêt pour la situation sociolinguistique du néerlandais belge avec Kas Deprez, un sociolinguiste belge précurseur, parti, lui aussi, bien trop tôt. Avec Jean, qui avait la bibliothèque complète des recherches de Kas, parce qu'ils s'envoyaient mutuellement leurs écrits et articles de presse, nous venions de finir d'une part nos articles dans La Bretagne Linguistique, co-écrits à la suite d'une présentation commune au GRELB, et d'autre part l'ouvrage ${ }^{3}$ qui rend hommage aux recherches de Kas qui posaient, depuis les années 80 , le pluricentrisme du néerlandais et l'existence d'un néerlandais belge standard, évolution qui émerge seulement actuellement. Le travail avec Jean était un énorme plaisir intellectuel, un pétillement de rires devant nos étonnements croisés, un engagement profond pour les locuteurs et leurs vécus, une saisie toute fine dans ses analyses sociolinguistiques et glottopolitiques ; j'ai beaucoup appris aux côtés de ce grand maitre, d'une gentillesse et d'une humilité rares dans nos universités. Et j'ai pu enfin, grâce aux réassurances de Jean, travailler sur ma propre langue maternelle et son évolution sociolinguistique, idée que je caressais depuis ma thèse et qu'il m'a permis de faire aboutir, tout à son incommensurable sens du partage.

Merciekes, Jean, ik zal je missen.

\section{Malo Morvan ${ }^{4}$ Inspe, Lyon 1}

J'ai été stupéfait d'apprendre la disparition de Jean Le Dû. Les dernières fois que je l'ai vu, en séminaire de La Bretagne Linguistique, à l'UBO à Brest, il donnait l'impression de n'avoir que 70 ans, et encore des années de recherche et d'échanges devant lui. Je l'avais d'abord connu par ses textes, dans le cadre de ma thèse, avec ce double statut de source primaire et secondaire, c'est-à-dire autant comme producteur d'un savoir de référence sur la matière bretonne que comme protagoniste engagé dans les débats que j'étudiais.

Lorsque j'ai participé pour la première fois au séminaire de La Bretagne Linguistique, et qu'il était présent, avec son camarade Yves Le Berre, j'étais impressionné, timide, peu confiant, et je n'ai pas vraiment osé aller l'aborder -d'autant que dans mon intervention de ce jour, il faisait partie du corpus de polémiques que je décortiquais ! Aujourd'hui, je regrette d'avoir si peu osé, en imaginant que j'aurais encore plusieurs années pour entamer une conversation à la Gentil'ho (le restaurant adjacent à l'UBO où l'on se réunit les jours de séminaire).

\footnotetext{
${ }^{3}$ Ledegen Gudrun \& Le Dû Jean, 2019, Pour un néerlandais de Belgique. Ecrits d'un précurseur. Hommage à Kas Deprez, L'Harmattan, Coll. « Espaces discursifs »

${ }^{4}$ Ce texte a circulé sur la liste RFS et a été repris sur le blog www.langue-bretonne.org, tenu par Fañch Broudic.
} 


\section{Des disciples du chanoine Falc'hun}

Délicat exercice que de restituer l'apport de Jean Le Dû, dans un contexte breton encore polémique, sans vouloir ranimer ici des débats qui seraient inappropriés en ces circonstances. Je m'y risque pourtant, estimant que sa contribution vaut la prise de risque.

Jean Le Dû et son inséparable compère Yves Le Berre étaient des disciples du chanoine François Falc'hun. Ce dernier avait proposé une analyse sociale et historique des évolutions géographiques du breton à partir de l'Atlas Linguistique de Basse-Bretagne de Pierre Le Roux (1927). Dans cette analyse, Falc'hun rapprochait davantage le breton du gaulois que d'un " celtique » atlantique, ce qui lui valut les foudres du milieu nationaliste défendant une différence radicale avec le français et un cousinage avec les iles et péninsules atlantiques. Ce conflit marquera également les prises de position de Jean Le Dû et d’Yves Le Berre.

\section{Des vigilances politiques}

Sans rentrer dans les détails de ces prises de position, sur la généalogie du breton, le lexique, ou l'orthographe, c'est avant tout un positionnement politique qui détermine les choix de Jean Le Dû : membre du PCF, il est vigilant à ce que le militantisme pour le breton ne reproduise pas un élitisme entre les intellectuel $\cdot l e \cdot s$, capables de comprendre les mots forgés à partir de racines celtiques, et la population rurale ne partageant pas cette culture étymologique, qui constituait à son époque la majorité du locutorat du breton.

Vigilant également à ce que la chasse aux « mots français » dans le breton ne reproduise pas un discours de purisme ou de repli sur soi, et à éviter une injonction à homogénéiser le breton (notamment dans sa graphie) qui ferait reproduire aux parlers locaux l'approche uniformisante que la langue bretonne avait pourtant subie.

Ces vigilances politiques ont été à la source de désaccords avec les linguistes et militants plutôt présents à Rennes, influencée tant par Roparz Hemon que par la sociolinguistique catalane, prônant une politique plus volontariste et unificatrice dans la promotion du breton, quitte à rompre avec son contexte initial de pratique, rural et oral, pour mettre en avant un nouveau locutorat, plus urbain et lettré. Alors que, pour certains, il s'agissait de «sauver le breton " avant tout, et toute méthode était bonne à cette fin, la volonté témoignée par Jean Le Dû de ne pas séparer la question sociolinguistique d'autres types de dominations politiques, et surtout de ne pas faire de la cause du breton la source de nouvelles discriminations sociales, me semble résonner éminemment avec les courants actuels qui étudient de manière (auto) critique les mouvements de revitalisation linguistique.

\section{Ancrer la langue bretonne dans le réel de ses locuteurs et locutrices}

Si Jean Le Dû a pu passer au-dessus de ces critiques, c'est, à mon sens, car il accordait plus d'importance aux bretonnant·e.s qu'à une «langue » prise pour elle-même. Ainsi disait-il, revenant de manière critique sur une journée à Rennes en 2003 consacrée aux « contacts de langue $\gg$ :

Ces journées accordent, avec raison, une grande place aux "contacts de langues ». C'est plutôt, selon nous, du contact entre locuteurs dont il faudrait parler, et le lieu du contact, c'est la conscience du locuteur.

Cette volonté de ne jamais aborder une «langue bretonne » hypostasiée, mais de toujours l'ancrer dans le réel de ses locuteurs et locutrices, et des contextes de pratique, qui marque toute sa méthodologie, me semble également tout à fait en prise avec les réflexions les plus contemporaines de la sociolinguistique, et l'importance qu'elles accordent à la pratique ethnographique. 
Une anecdote à ce sujet : avant d'être lui-même linguiste, il avait été, en 1958, informateur pour l'enquête menée par Kenneth Jackson sur le breton de Plougrescant, voici ce qu'il en dit dans le paratexte introductif à son dictionnaire sur le breton de Plougrescant :

Il [K.H. Jackson] avait établi une liste de mots dont il souhaitait connaitre la réalisation, ce qui l'a entrainé à me demander d'en prononcer certains qui m'étaient inconnus, comme amezeg "voisin» ou mell «grand»... procédé qui n'avait pas manqué de me surprendre.

Il n'est sans doute pas fréquent que l'informateur se fasse à son tour descripteur. La comparaison entre les deux études pourrait alimenter une réflexion sur la linguistique de terrain, sur les différences d'approches et de résultats entre deux descriptions, dont l'une, élaborée dans l'urgence, est faite en peu de temps par un savant extérieur au milieu dont il étudie la langue, tandis que l'autre est faite de l'intérieur et sur la longue durée.

\section{Un dictionnaire, un atlas...}

Son dictionnaire sur le trégorrois à Plougrescant, justement, une des quelques œuvres monumentales qu'il a menées à bien (2012, chez Emgleo Breiz). Ouvrage en deux tomes, de 1038 pages, au sujet d'une commune qui comptait 1284 habitant·e·s en 2012, année de sa parution. Soit presque une page par habitant·e ! Ce dictionnaire, élaboré à partir de sa thèse d'État datant de 1978, et patiemment complété depuis, explicite la seconde grande source d'inspiration de Jean Le Dû, après François Falc'hun : sa mère, Mai Maguer, à laquelle il rend un vibrant hommage dans son ouvrage.

Une autre de ses importantes contributions, le Nouvel Atlas Linguistique de Basse-Bretagne, paru en 2001, en 2 volumes et 600 cartes. À ma connaissance, il n'y a pas eu de recherche de faite pour voir si ce Nouvel Atlas offrait de nouveaux éclairages aux hypothèses faites par Falc'hun sur l'Atlas de 1927 (peut-être qu'il y en a et que je ne les connais pas).

\section{Le dialogue avec d'autres courants de la sociolinguistique}

Jean Le Dû et Yves Le Berre ont fait partie des rares sociolinguistes bretons à tenter de proposer une conceptualisation qui, à partir du terrain breton, permette de dialoguer avec d'autres courants de la sociolinguistique, comme en témoignent les colloques organisés en 1994 et en 1997, regroupant des sociolinguistes de tous horizons venus discuter de la pertinence du modèle « badume/standard/norme » et comparer leurs approches.

Leurs deux contributions théoriques principales résident dans cette typologie «badume/standard/norme » et dans la distinction entre les sphères «paritaire » et « disparitaire » de la langue.

Le terme « badume », signifiant «par chez moi » en breton, est défini ainsi :

Le badume a comme principal caractère d'être un parler familier, identitaire, qui, selon ses locuteurs, ne s'enseigne pas, mais s'acquiert naturellement, par imprégnation, comme l'air qu'on respire. D'où l'amusement, parfois l'agacement et toujours la gêne des locuteurs du breton hérité quand des apprentis-bretonnants s'adressent à eux en breton. On les félicitera bien sûr de leurs efforts. "Toi, tu parles mieux que nous », mais on ne les encouragera guère à aller plus loin. Si l'on est réticent à utiliser le badume devant des étrangers, ça n'est pas en raison d'un quelconque complexe d'infériorité, mais c'est parce qu'il y a des choses qui ne se disent que dans un cadre intime et pas en public, ce cadre intime se réduisant de plus en plus, en raison des bouleversements linguistiques de ces dernières décennies, au voisinage, à la maisonnée, voire à une partie de la classe d'âge. 
Le badume s'inscrit entièrement dans l'oralité, ce qui explique la réticence de ses locuteurs à tout apprentissage de l'écriture. Comme le prestige est du côté de l'écrit, ses usagers font cependant mine d'admirer ceux qui s'essaient à transcrire le badume, c'est-à-dire vont contre la normalité, en commentant : "Le breton, c'est dur à écrire!». Des règles strictes existent dans le badume, qui se sont établies en opposition avec ce que disent les voisins : eux, ils disent oaran ked (pour 'je ne sais pas'), mais ici on dit n'ouzon ked. On corrige les enfants - quel type de fautes peuvent-ils faire en fait? -, non pour se conformer à un idéal externe, mais pour respecter un consensus local. N'en est-il pas de même pour l'habillement, la pratique religieuse, voire la façon de cuire les pommes de terre? [...].

Dans la mesure où la société est stable, le badume évolue peu, et reste même très conservateur. On persiste à compter en réaux, livres et écus quand le franc les a remplacés depuis longtemps. Mais tout bouleversement social se reflètera rapidement dans le badume. Ainsi en est-il de la disparition quasi totale dans les mémoires $d u$ vocabulaire des métiers tombés en désuétude : instruments aratoires, outils du charron, techniques du sabotier... Le badume est lié à une société paysanne qui est de nos jours en phase d'extinction finale. (dans La Bretagne Linguistique 10 ; 1996, 7-25)

Peu de sociolinguistes peuvent s'enorgueillir de ce que leurs concepts ont servi à nommer des groupes de musique (si l'on fait exception de la Compagnie créole...). Or il existe bien le Badume's Band (le groupe de par chez moi), groupe breton qui joue bien entendu... de la musique éthiopienne. En attendant les polynomies corses, ou le diglossic quatuor $(\mathrm{H}) /$ quartet (L)...

\section{Théoriser une approche moins conflictuelle des situations de diglossie}

L'opposition entre sphères ou registres paritaire et disparitaire vise à prolonger les débats sur la diglossie. Au-delà d'une définition qui peut sembler reprendre classiquement la bipartition H/L chez Ferguson

Le registre de la parité est le versant chaud de cette dualité. [...] C'est le langage de l'intimité, de la fraternité, de la solidarité, de la familiarité, de l'égalité, de la liberté de l'individu ou du groupe dans l'ensemble social. Son rayon de communication est géographiquement ou socialement restreint et limité à l'oralité. [...] Le registre de la disparité en est symétriquement le versant froid. C'est celui de l'autorité, de l'officialité, de la représentativité, de la formalité, de la régularité, de l'institution. Sa capacité de communication est universelle (dans l'ensemble des locuteurs de la langue) et fondée sur l'écriture. (ibid., p. 20).

Il s'agit pour les deux auteurs de théoriser une approche moins conflictuelle et plus irénique des situations de diglossie, qui explique la transition progressive du breton au français, en défendant notamment la thèse (comme le fait également Fañch Broudic dans La pratique du breton de l'Ancien Régime à nos jours, PUR, 1995) selon laquelle la diminution de pratique du breton fut en partie volontaire, liée à la disparition d'une société paysanne traditionnelle et à l'adaptation à l'économie de marché, et donc pas uniquement à une politique linguistique française volontariste. Là encore, marxistes qu'ils étaient, en intégrant très tôt la question de l'intérêt économique dans l'analyse des attitudes linguistiques, les fondateurs du GRELB (Groupe de Recherche sur l'Économie Linguistique de la Bretagne) me semblaient avoir pressenti des courants de recherche bien actuels. 


\section{Toujours ouvert aux nouvelles informations}

Au-delà de l'œuvre, les aperçus que j'ai eus de la personne, en séminaire à Brest, me montraient un homme doux, à la pensée vive, volontiers goguenard, mais jamais médisant. Malgré la position de sommité que lui et Yves Le Berre avaient acquise, il était simple et humble dans sa présentation et son rapport aux autres. En particulier, alors qu'il aurait pu se reposer sur les lauriers de ses contributions massives, il semblait toujours ouvert aux nouvelles informations, approches, et théories, y compris lorsqu'elles bousculaient un peu les acquis qu'il avait établis, avec d'autres, il y a quelques décennies — belle humilité, pas si fréquente dans nos milieux.

Tout cela rendait d'autant plus infondées les réserves que j'avais pour entrer avec lui dans un échange plus direct, et me le fait d'autant plus regretter aujourd'hui. Alors tant qu'à lui rendre hommage aujourd'hui, je voudrais dire aux gens de mon âge : si vous avez autour de vous quelqu'un comme Jean Le Dû, osez donc aller lui parler tant qu'il est temps, même si cette personne semble n'avoir que 70 ans !

Je ne sais pas s'il existe un paradis pour les membres du PCF ni c'est le genre de lieu qui les attire, c'est pourquoi je lui souhaite au moins de continuer à résonner dans nos prochaines discussions de sociolinguistique.

Avec une pensée pour sa famille, ainsi que son compère Yves Le Berre, et ses collègues et anciens collègues de l'UBO.

\section{René Tabouret-Keller, compagnon d'Andrée}

Chers amis «par compagnonnage» (comme on dit «par alliance»), je suis l'époux d'Andrée Tabouret-Keller.

Les nonagénaires s'en vont. Frédéric François, Marcellesi, Chaudenson, Le Dû, ... ; Andrée voit partir, plus que des collègues, ses «camarades scientifiques » et amis tel Jean Le Dû. Qu'avaient-ils en commun ? ce que Malo Morvan a très heureusement souligné : s'intéresser au locuteur dans son humanité, déplacer le regard du linguiste, porter l'attention sur l'acte de parler et sur les «parlers » (le labo d'Andrée était intitulé «Langage, dimensions sociales et incidences subjectives »). Leurs terrains offraient des situations linguistiques complexes, porteuses de dynamiques langagières souvent socialement conflictuelles (Corse, Bretagne, Alsace, pays Basque, Québec, Occitanie,... ), impliquaient des recherches à portée politique (le « bilinguisme » a valu à Andrée, dans les années 70, d'être consultée en Catalogne au moment de la mort de Franco, puis par le secrétariat à l'éducation du Gouvernement Basque, ou, avec Le Page, par celui du Bélize accédant à l'indépendance, ou plus tard par l'université chinoise de Hong-Kong), recherches riches de questionnements sur la vie des langues et celle des gens qui les parlent (les créoles et la Caraïbe, toute une histoire !).

Familier d'un autre milieu (travaux publics et urbanisme) mais témoin rapproché et intéressé, j'évoque ici ce que j'ai perçu (à travers les retours de terrain ou de colloque et les articles d'ATK) des façons qu'ils ont eues de vivre leur aventure de chercheur : les filiations sans servitude (Martinet maitre de Weinreich maitre de Fishman et de Labov, et qui introduit Andrée auprès de Weinreich dans les années 60); l'emploi rigoureux du savoir linguistique qui permettait d'éviter les biais idéologiques ; le souci d'éveiller plus que de transmettre y compris en sortant des champs balisés comme ils l'avaient fait eux-mêmes; les "croisements » avec d'autres pensées du sujet dans le langage et dans l'institution (Lacan, Foucault,..) et l'engagement fraternel sur des points chauds (adhésion au PCF, enseignement du plurilinguisme en Algérie). Au-delà de la trace que chacun a laissée et qui vous est familière, j'ai voulu évoquer la proximité amicale et intellectuelle de vos « anciens ». 


\section{Mannaig Thomas et Nelly Blanchard co-directrices de La Bretagne linguistique, pour le GRELB (CRBC)}

«Pa vije klewed ar hloh vije gouveed vije eur maro, ha vije goulled: "piou zo maro?" emê. Ha vije klewed gand an hini vije bed er bourk / Quand on entendait la cloche, on savait qu'il y avait un mort, et on demandait : "qui est mort ?" disait-on. Et celui qui était allé au bourg nous l'apprenait », racontait Anne-Marie, une informatrice plougrescantaise privilégiée de Jean Le Dû.

Nous souhaitons ici rendre hommage à Jean dont les travaux de sociolinguistique et de dialectologie sont de véritables monuments scientifiques, tant par leur ampleur que par leur importance conceptuelle. En 1985, avec Yves Le Berre, ils publiaient le premier numéro de la revue La Bretagne Linguistique issu des travaux du Groupe de recherche sur l'économie linguistique de la Bretagne (GRELB) qu'ils avaient fondé l'année précédente. Initiateurs d'un projet de recherche au long cours, ils ont posé des bases scientifiques, épistémologiques et humaines solides qui lui ont permis de se poursuivre au-delà de leur temps de carrière et que nous avons le plaisir de continuer aujourd'hui. Par fidélité pour la jeune génération et parce qu'il poursuivait ses travaux sur le breton, mais également sur de nombreuses autres langues (gaëlique, créole, flamand etc.), Jean continuait de participer et d'intervenir régulièrement au séminaire de « La Bretagne Linguistique ».

Sociolinguiste et dialectologue reconnu nationalement et internationalement, Jean avait une connaissance très fine du breton dans sa variation, comme en témoigne, notamment, le Nouvel Atlas Linguistique de la Basse-Bretagne qu'il avait publié en 2001, mais également son implication dans l'Atlas Linguarum Europae ou l'Atlas Linguistique Roman. Pionnier de la sociolinguistique du breton avec Yves Le Berre, le cheminement de leurs réflexions dans ce domaine est paru en 2019 sous la forme d'un recueil d'articles intitulé Métamorphoses. Trente ans de sociolinguistique à Brest (Brest, éditions du CRBC, coll. "Lire/Relire»). La bibliographie de Jean Le Dû est également accessible sur le site du Centre de recherche bretonne et celtique.

Le GRELB souhaite par ce message exprimer sa tristesse suite à la perte d'un grand chercheur, mais également d'un collègue et ami. 


\section{GLOTTOPOL}

Revue de sociolinguistique en ligne

Comité de rédaction : Michaël Abecassis, Salih Akin, Sophie Babault, Claude Caitucoli, Véronique Castellotti, Régine Delamotte, Robert Fournier, Stéphanie Galligani, Emmanuelle Huver, Normand Labrie, Foued Laroussi, Benoit Leblanc, Fabienne Leconte, Gudrun Ledegen, Danièle Moore, Clara Mortamet, Alioune Ndao, Isabelle Pierozak, Gisèle Prignitz.

Rédactrice en chef : Clara Mortamet.

Comité scientifique : Claudine Bavoux, Michel Beniamino, Jacqueline Billiez, Philippe Blanchet, Pierre Bouchard, Ahmed Boukous, Pierre Dumont, Jean-Michel Eloy, Françoise Gadet, Monica Heller, Caroline Juilliard, Jean-Marie Klinkenberg, Jean Le Dû (†), Marinette Matthey, Jacques Maurais, Marie-Louise Moreau, Robert Nicolaï, Didier de Robillard, Paul Siblot, Claude Truchot, Daniel Véronique.

\section{Comité de lecture pour ce numéro :}

Salih Akin, Carmen Alén Garabato, Sophie Babault, Philippe Blanchet, Henri Boyer, Véronique Castellotti, Marisa Cavalli, Jean-François De Pietro, Didier de Robillard, Alain Di Meglio, Ksenija Djordjevic, Jean Michel Eloy, Pascale Erahrt, Véronique Fillol, Monica Heller, Robert Fournier, Normand Labrie, Hervé Lieutard, Jean Le Dû (†), Marinette Matthey.

http://glottopol.univ-rouen.fr

ISSN : 1769-7425 